\title{
A Photovoltaic Module Diagnostic Setup for Lock-IN Electroluminescence Imaging
}

Parikh, Harsh Rajesh; Spataru, Sergiu Viorel; Séra, Dezso; Mantel, Claire; A. dos Reis Benatto, Gisele; Poulsen, Peter; Frederiksen, Kenn H. B.; Forchhammer, Søren; Vedde, Jan

\section{Published in:}

Proceedings of 46th leee Photovoltaic Specialists Conference (pvsc 46)

Link to article, DOI:

10.1109/PVSC40753.2019.8981255

Publication date:

2020

Document Version

Peer reviewed version

Link back to DTU Orbit

Citation (APA):

Parikh, H. R., Spataru, S. V., Séra, D., Mantel, C., A. dos Reis Benatto, G., Poulsen, P., Frederiksen, K. H. B., Forchhammer, S., \& Vedde, J. (2020). A Photovoltaic Module Diagnostic Setup for Lock-IN Electroluminescence Imaging. In Proceedings of 46th leee Photovoltaic Specialists Conference (pvsc 46) IEEE.

https://doi.org/10.1109/PVSC40753.2019.8981255

\section{General rights}

Copyright and moral rights for the publications made accessible in the public portal are retained by the authors and/or other copyright owners and it is a condition of accessing publications that users recognise and abide by the legal requirements associated with these rights.

- Users may download and print one copy of any publication from the public portal for the purpose of private study or research.

- You may not further distribute the material or use it for any profit-making activity or commercial gain

- You may freely distribute the URL identifying the publication in the public portal 


\title{
A PHOTOVOLTAIC MODULE DIAGNOSTIC SETUP FOR LOCK-IN ELECTROLUMINESCENCE IMAGING
}

\author{
Harsh R. Parikh ${ }^{1}$, Sergiu Spataru ${ }^{1}$, Dezso Sera ${ }^{1}$, Gisele A. dos Reis Benatto ${ }^{2}$, Peter B. Poulsen ${ }^{2}$, \\ Claire Mantel ${ }^{2}$ Soren Forchhammer ${ }^{2}$, Michael Larsen ${ }^{3}$, Kenn H. B. Frederiksen ${ }^{4}$ and Jan Vedde ${ }^{5}$ \\ ${ }^{1}$ Aalborg University, Department of Energy Technology, 9220, Aalborg, Denmark \\ ${ }^{2}$ Technical University of Denmark, Department of Photonics Engineering, 4000, Roskilde, Denmark \\ ${ }^{3}$ Sky-Watch A/S, stre Alle 6 Stvring, 9530, Nordjylland, Denmark \\ ${ }^{4}$ Kenergy, Grnningen 43, 8700, Horsens, Denmark \\ ${ }^{5}$ SiCon Silicon and PV consulting, J N Vinthersvej 5, 3460, Birkerd, Denmark
}

\begin{abstract}
Electroluminescence (EL) imaging and infrared (IRT) thermography techniques have become indispensable tools in recent years for health diagnostic of photovoltaic modules in solar industry application. We propose a diagnostic setup, which performs lock-in EL for accurate analysis of different types of faults occurring in a solar module. The setup is built around a high-speed SWIR camera, which can acquire images at very short integration time $(1 \mu \mathrm{s})$ and high frame rate $(301 \mathrm{fps})$. In addition, a state-of-the-art imaging chamber allows for introducing controlled levels of ambient light noise for developing new light noise removal methods, rotation of panel frame in 3 axes plane for developing perspective distortion correction techniques. The paper also gives an insight of different system and communication delays that affects the performance of overall EL lock-in imaging system integration. The purpose of the diagnostic setup is to support research in PV failure quantification through EL imaging, which can also be useful for aerial drone imaging of PV plants.
\end{abstract}

Index Terms-EL imaging, lock-in technique, ambient noise level, perspective distortion, lock-in measurement procedure, system delays.

\section{INTRODUCTION}

Photo-voltaic (PV) module failures are ubiquitous, they can develop due to innumerable reasons, and in different stages of the module lifetime and thus detection and quantification of these faults and its severity have become important. Thus, EL imaging has become an imperative diagnostic tool for PV modules, allowing identification of a wide range of solar cell failure [1]. Most often, EL imaging is used as a qualitative diagnostic tool however; it presents significant potential to quantify the magnitude or severity of degradation and defects through visual image analysis methods.

Lock-in technique has become a standard technique since several years for investigating shunting phenomena in solar module research. The working principle of this technique is; power dissipated in the device under test (DUT) is periodically amplitude-modulated, and an InGaAs camera images the surface of PV modules. The camera runs at a certain frame rate and the generated images are digitally processed according to the lock-in principle for camera based systems which is described in [2]. The implementation of lock-in technique eliminates the ambient light noise by periodically modulating the input signal and averaging it over a set number of periods derived from the frame rate and the lock- in frequency [3].

The aim of this paper is to describe a versatile experimental setup, which can acquire images through EL lock-in (ELLI). Since the SWIR camera is capable of recording images at a frame rate of 301fps [4], it would be interesting to see if the system integration allows imaging the panel at the maximum camera frame rate with all the system delays taking place during synchronization. The reason for choosing a camera with high integration time is because it allows eliminating the constant changing weather condition factor while performing outdoor EL measurements ensuring that the signal images and background (BG) images are taken under same ambient conditions.

Some of the listed experiments which the setup would be capable of performing are:

- Evaluating the impact of different setting factors i.e. (lock-in frequency, different levels of ambient light noise and varying bias) that can affect image analysis and PV diagnostic while performing experiments.

- An option of performing exposure sweep in the developed GUI while executing PV modulation is also designed.

- ELLI/sunlight for failure analysis and its effect on PV diagnostics.

- Finally, experiments will be carried out at different tilt angles for perspective distortion correction and recognizing the panel frame as displayed in Fig.1.

It is important to have a setup that is able to perform experiments where camera will not always be perpendicular to the (DUT), which will be the case during aerial drone imaging of PV plants for diagnostics, which is the ultimate goal of this research and designing the setup.

\section{METHODOLOGY}

\section{A. Hardware Setup}

Fig.2 displays the hardware experimental setup that is used for carrying out experiments through EL imaging technique. The setup consists of a (Solvis 36-150 E) mono-crystalline silicon PV module (DUT), which is connected to a bidirectional Kepco BOP 1000 MG power supply to feed and modulate 


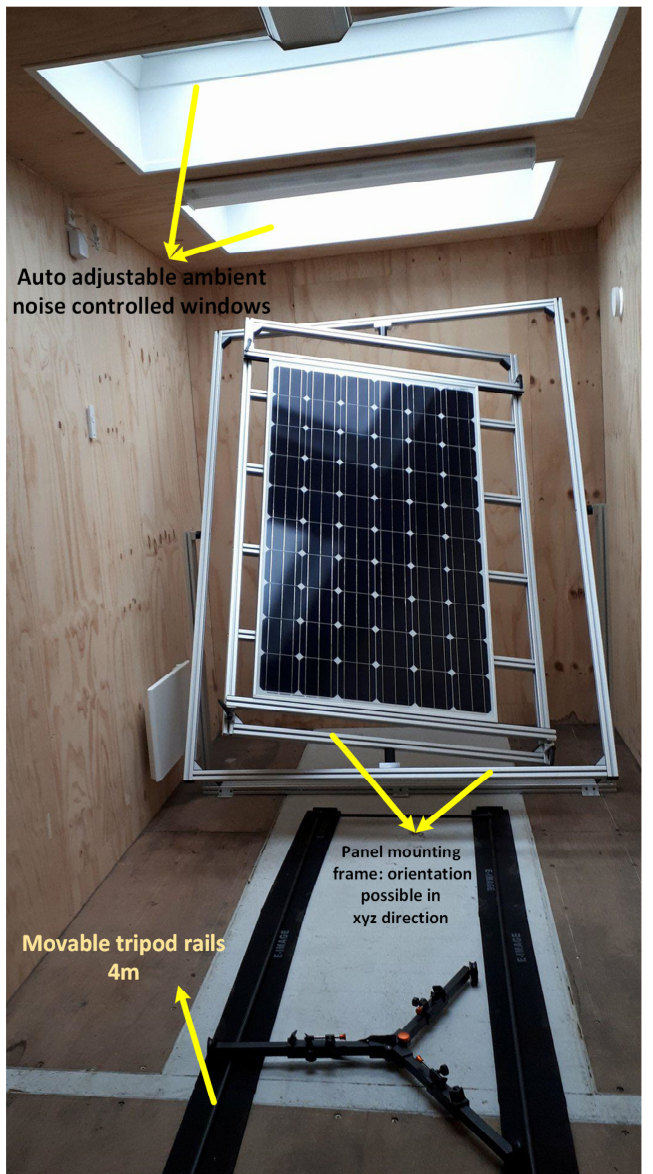

Fig. 1. Indoor imaging lab design

the current through the module during ELLI. Goldeye CL033 high-speed SWIR is used for carrying out lock-in EL inspections. The advantage of this power supply is that it is capable of performing four- quadrant operation measurements [5]. This power supply was chosen because of its bidirectional operation and relatively fast rise and fall time $(200 \mu \mathrm{s})$. A high frame-rate SWIR camera provides an advantage of imaging at high resolution with very short exposure time inferring in a long inspection time, which can be considered as an asset for UAV applications and especially for large PV plant inspection.

\section{B. Lock-in measurement procedure}

The lock-in principle technique used for this research is generation of a modulated square wave voltage signal with a specific pulse period chosen as a multiple of the camera acquisition period as shown in Fig.3. Over here we choose to sample with at least two samples per period while performing ELLI, thus satisying the sampling theorem [6] compared to what can be chosen for performing IRT [7]. Hence, the camera acquisition frame rate needs to be at least twice of your PV modulation frequency. Over here, a camera is running with a certain frame rate capturing the induced photons in a PV module and converting into an image containing the sum

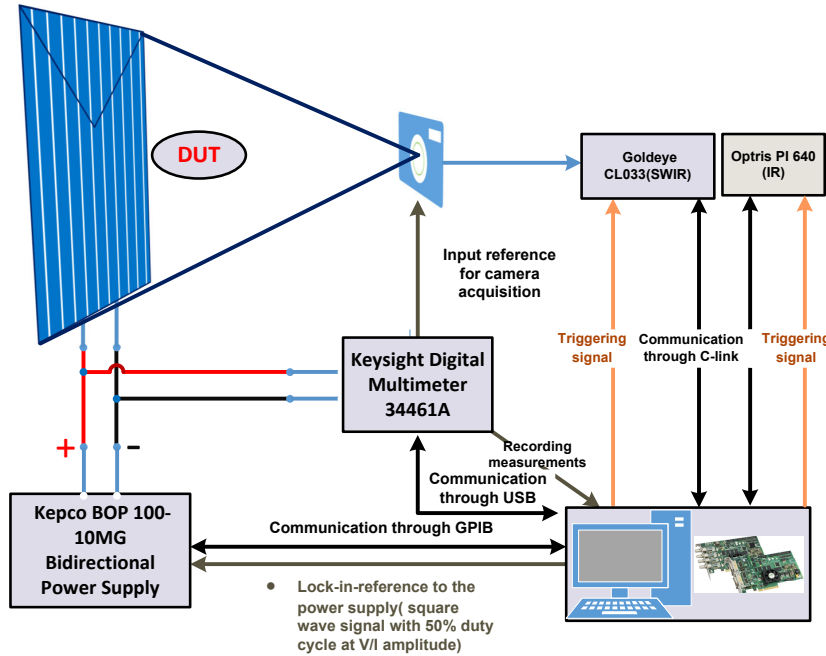

Fig. 2. Hardware experimental setup with system configuration for automated EL lock-in(ELLI) imaging.

of pixel information. For camera-based systems, the exposure time equals the total image acquisition time of the experiment and should not be complicated with the frame integration time, which infers as the time when the electronic shutter of the camera is open.

In practice, one also has to consider the delays affecting the system and hence the camera acquisition frame-rate is decided based on the PV modulation and the delays. For fixed sampling rate $f_{s}$, the number of samples per lock-in period $n$ gives the lock-in frequency shown in (1):

$$
f_{\text {lock-in }}=\frac{f_{s}}{n}
$$

Firstly, forward bias applied to the PV consisting of a programmed square wave $\mathrm{DC}$ voltage signal, pulsing between $0 \mathrm{~V}$ and $V_{o c}+50 \mathrm{~V}$, where, $V_{o c}$ is the open circuit voltage under standard test conditions (STC). Since the PSU was controlled in voltage mode, corresponding current limits were set to 0 $\mathrm{A}$ and STC $I_{s c}$ (approximately 8A). The pulsing frequency range of the excitation reference lock-in signal is defined by the power supply, which in this case can vary from 0.02 $1000 \mathrm{~Hz}$ [8] and also by the camera acquisition frame rate. The power supply-PC synchronization is done through GPIB communication. The delay between the reference given to the power supply for generating the modulating signal and its actual signal generation cannot be measured physically. Hence, a high precision Digital Multimeter (DMM) is used for recording the measurements of the lock-in signal. This is necessary for synchronizing the triggering of the DMM with the lock-in reference signal for detecting its first high bias state as shown in Fig.3. The communication between the DMM and the PC is done through USB connection. Detection of the first high bias and low bias calculation is recorded through the data values logged by the DMM. The trigger delay for the DMM i.e. the occurrence of trigger and the first measurement is $160 \mu \mathrm{s}$. This delay needs to be considered 
while synchronizing the DMM and power supply with the PC.

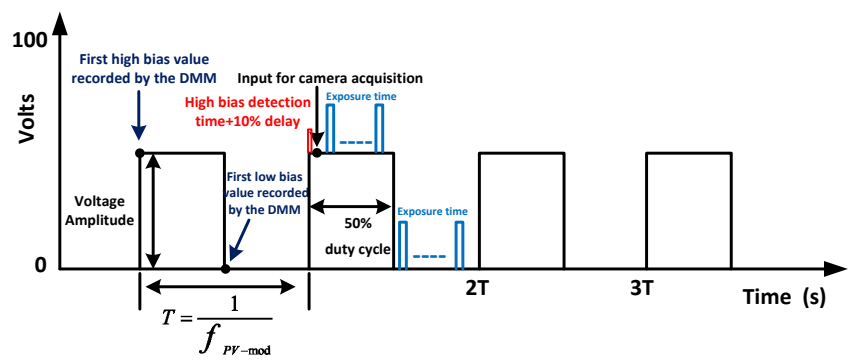

Fig. 3. Graphical representation of a square wave-modulating signal displaying the synchronization of the DMM and camera with the lock- in reference.

An integration time of 1 power line cycles (PLC) with a $20 \mathrm{~ms}$ resolution is chosen [8]. The time it takes for the power supply to reach from one bias state to another is in the range of (30-60) $\mu s$. Thus for the first high and low bias detection, a tolerance of $10 \%$ of the bias value of the generated lock-in reference signal is considered to make sure that the camera acquisition starts at high bias peak. Since, the lock-in reference was generated with $50 \%$ duty cycle; the time for detecting the first rising and falling edge of the excitation signal remains the same. The purpose of low bias calculation is calculated in order to validate that the synchronization done for recording the first high bias is accurate and the algorithm is valid.

Once the DMM records the first high and low bias of the signal, a delay of one modulation period is given as an input to the camera to start acquiring images. The camera starts acquiring images continuously once the acquisition event starts and will acquire images defined by the acquisition frame count. The whole integrated system measurement procedure flowchart is displayed in Fig. 4

\section{System delays}

Fig. 5 displays the modulating reference signal sent to the power supply for executing EL lock-in. It is important to add the tolerance and the DMM trigger occurence delay $(160 \mu s)$ in the system before camera triggering is executed to make sure that the camera acquires images during its desired state i.e. $I_{s c}$ bias and no bias. The fall time during the switching states increases with the increase in PV modulation frequency. At $10 \mathrm{~Hz}$ the settling time of the input voltage from $I_{s c}$ bias to no bias is less than $1 \mathrm{~ms}$ as shown in the figure on the left and it increase to $2.2 \mathrm{~ms}$ with $60 \mathrm{~Hz}$.

This difference of $2.2 \mathrm{~ms}$ remains constant at higher PV modulation frequency. While, designing a system, one needs to take into account the limitation of the power supply. The rated rise and fall time for the voltage input channel is $(200 / 250) \mu s$. Thus, it is necessary for the proper ELLI execution to take into account the aforementioned delays for the intended camera acquisition performance.

The delays caused by the settling time, DMM triggering for

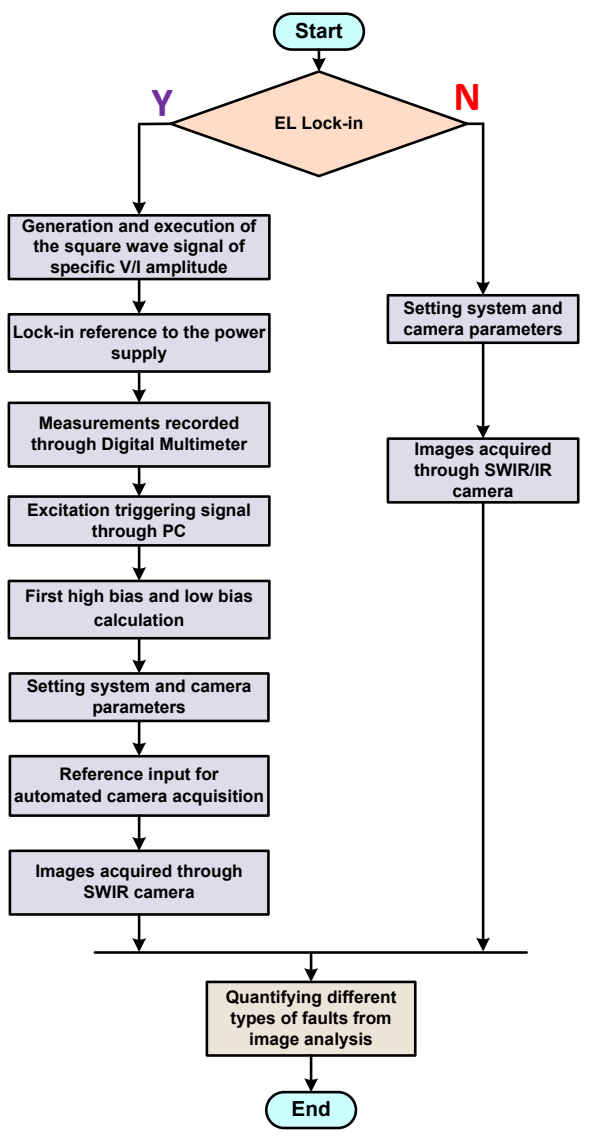

Fig. 4. Structural flowchart of the implemented measurement procedure
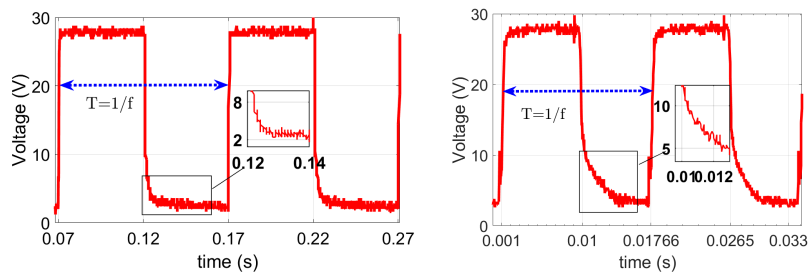

Fig. 5. Settling time of the modulating reference signal with $10 \mathrm{~Hz}$ (left plot) and $60 \mathrm{~Hz}$ (right plot) for EL lock-in (ELLI) implementation

recording the modulating voltage values and camera image readout play a vital role in choosing the appropriate camera frame-rate and exposure time. The camera acquisition framerate is chosen to be atleast twice that of the PV modulation frequency. The inter dependency between the exposure time, camera acquisition frame-rate and PV modulation frequency is highlighted in the equations presented in (2) and (3). The maximum frame-rate which was managed to be achieved keeping in mind an optimal exposure time for obtaining a high contrast EL image without it getting underexposed was $120 \mathrm{~Hz}$.

$$
f_{\text {frame-rate }}=2 \cdot f_{P V}
$$




\section{Camera software control}

A Goldeye CL-033 EL SWIR camera is used for camera acquisition. The camera is connected through a PC via an Xcelera frame grabber (FG) camera link board. The hardware connection and communication between the camera and the FG board is completed using a camera link cable. An interface is created where we first verify if the camera is properly connected and synchronized to the PC through which it can be controlled. It is to be noted that the total registered exposure time for image acquisition is sum of exposure start delay and the image readout time with exposure start delay being $1 \mu \mathrm{s}$ and the frame readout for transferring of image data is $26 \mu \mathrm{s}$. Hence, while choosing an optimal exposure time for imaging a storage time of $26 \mu \mathrm{s}$ needs to be added to the desired set exposure time [4]. The statistical equation governing the image acquisition can given by (3). The exposure time is chosen depending on the camera acquisition frame rate and the correlation formula between the two is:

$$
\begin{gathered}
T_{\text {exp }} \leq \frac{1}{f_{\text {frame-rate }}} \\
I_{\text {acq }}=T+0.05 \cdot T+T_{\text {exp }}+T_{\text {img-readout }}
\end{gathered}
$$

\section{E. Panel Orientation control}

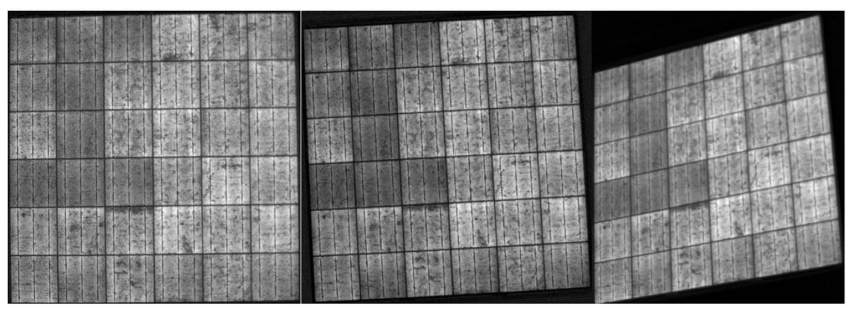

Fig. 6. EL images with different angles of perspective distortion (PD).

It is possible to image the panels at different desired tilt angles by displacing it in 2 axes(xy) and 3 axes(xyz) direction plane with redundancy. The structure can measure a 72-cell panel with camera to panel distance being $450 \mathrm{cms}$ and a $60-$ cell module with camera-panel distance being $300 \mathrm{cms}$ with a horizontal rotation from: $(-60: 60)^{\circ}$ with a step size of $1^{\circ}$ covering a range of $120^{\circ}$ and the same could be performed for vertical rotation as well. EL lock-in (ELLI) measurements with different angles of perspective distortion (PD) under controlled and reproducible manner is displayed in Fig. 5 where, camera is not perpendicular to DUT. Another example of image acquisition highlighting the shading of the cells while the drone integrated imaging system is perpendicular to the imaging panel, as shown in Fig. 6 and to avoid that, we might have to acquire images at a specific perspective distorted angle. With aerial imaging as the main goal, a structure was built which could replicate different test scenarios where we can intentionally distort the panel orientation and look for practical solutions of perspective distortion correction (PDC) described in [9] [10].The measurements can be taken from a stationary position as well as can be converted into a movable test setup with the help of mounting rails as displayed in Fig. 1. The figure displays an effect on the

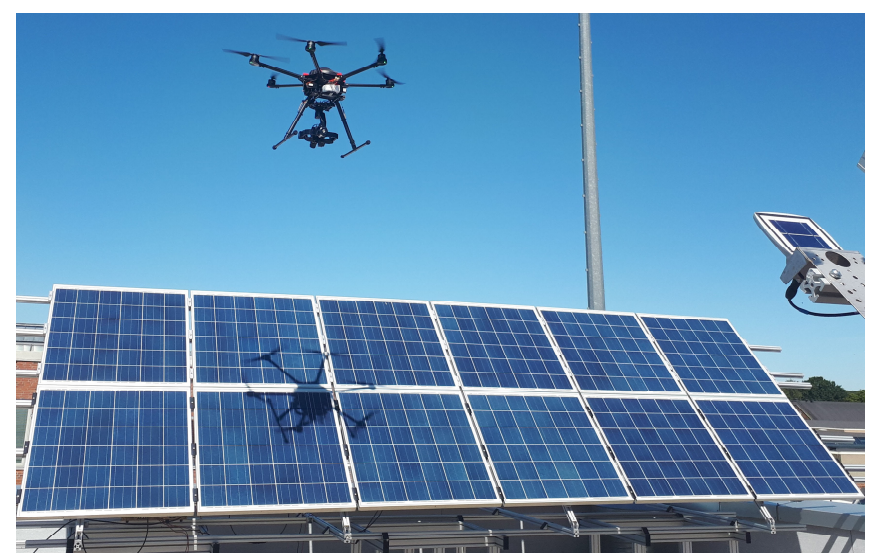

Fig. 7. Aerial drone based EL measurments shading the panel while performing outdoor measurements when camera is approximately perpendicular to the imaging panel.

intensity distribution of an acquired EL lock-in (ELLI) image with and without perspective distortion (PD). This can prove to be an essential characteristic to investigate, as it would also effect the statistical parameters while quantifying different types of faults.

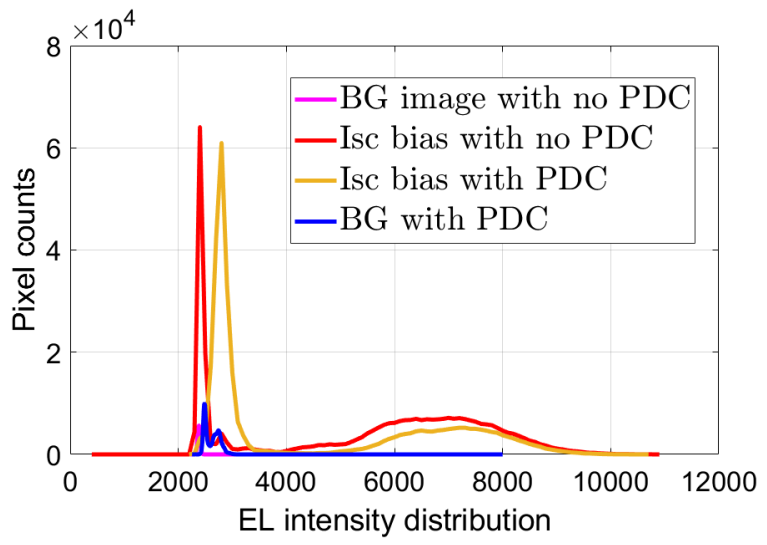

Fig. 8. Intensity distribution of the EL images with and without perspective distortion (PD).

In aerial imaging another problem which is necessary to investigate is the motion effect on the image quality. The 'motion effect' affects when we need to have several images averaged and subtracted due to sub-pixel level displacement resulting in a compromised image quality for cell fracture and defects detection. The image displaying the effect of motion (right side) and its resulting motion compensated image (left side) is shown in Fig. 8. For compensating the effects of motion on the acquired measurements, firstly, in each frame, the panel is detected and roughly segmented from the rest of the elements in the field of view. That allows compensating the motion between different frames and registering across all images of the sequence which is explained in [11]. 


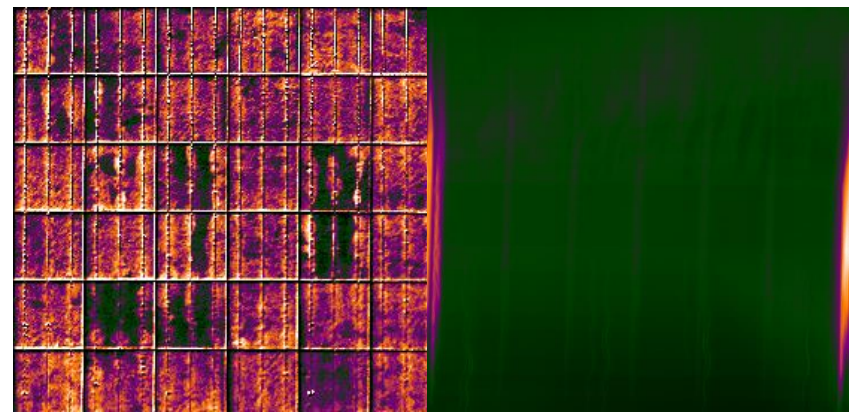

Fig. 9. EL measurements with and without motion compensation. left: resulting image after applying motion compensation algorithm; right:image acquired with moving camera

\section{F. Camera exposure sweep}

An option of performing exposure sweep in the developed GUI while executing PV modulation is also designed as shown in Fig.9. The idea behind designing this feature is to determine the optimal exposure time for EL images, in respect to maximizing the camera sensitivity range and knowing the saturation limit. Having an idea of the system experimental parameters before performing aerial inspection would result into shorter and better quality EL measurement process. It will also help in obtaining accurate images for qualitative and quantitative evaluation of PV module failures for determining the health status of the acquired PV panels.

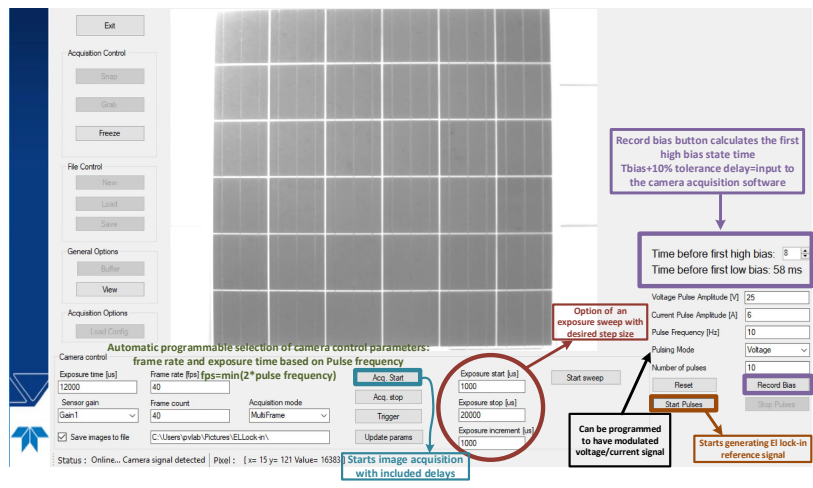

Fig. 10. GUI software of the implemented EL lock-in integrated system.

\section{G. Controlled ambient noise}

It is possible to introduce ambient noise on to the panels in a controlled and quantifiable manner. This will help investigating the advantage and the extent to which, different image enhancement methods that can be useful in eliminating the noise [12], and to estimate the effect of the background noise on the imaging process and to better understand the noise characteristics towards an InGaAs detector and develop image post processing strategies accordingly [13]. The effect of different ambient noise level on an EL image visualization is depicted in Fig.10. The measurements were taken just after the solar noon had passed at around 13:00 pm on a relatively bright sunny day for introducing maximum sunlight possible inside the chamber. A camera with high integration time was chosen since it allows eliminating constant changing weather condition factor while performing outdoor EL measurements ensuring that the signal images (EL) and background (BG) images are taken under same ambient conditions. Once the measurement procedure is completed,

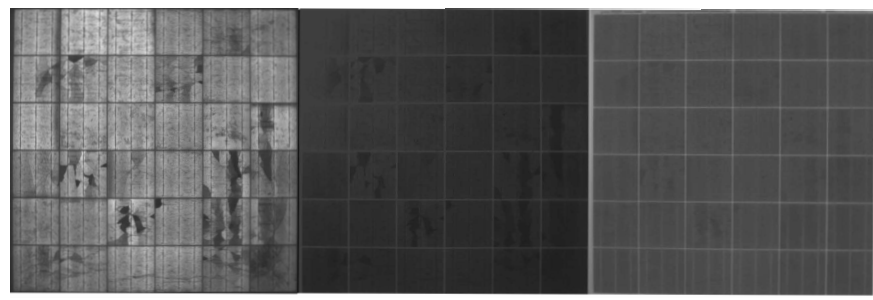

Fig. 11. Effect of different levels of ambient noise on EL original image. Dark measurement with no noise (left); $50 \%$ of ambient light noise incident on panel (center); maximum possible amount of ambient light noise (right).

an averaging and subtraction image enhancement technique is used to reduce the ambient noise level. As averaging several pictures is the most common way to minimize noise from images, we perform it to enhance image quality [12]. The image processing included taking the difference between the average of light (with EL signal) and dark images (background/unpowered EL image). Signal-to-noise ratio (SNR) of the following images is also analysed and is reflected by improving the quality of images for the purpose of more accurate failure detection as shown in Fig.10 [12].

\section{SUMmARY AND OUTLOOK}

EL Lock-in along with its final algorithm is implemented on a healthy and fault inflicted module for carrying out PV diagnostics. The setup was built with the intention of carrying out experimental work and investigating the different technical problems like the camera inclination to DUT, different levels of light noise levels affecting the recorded measurements for SNR computation, effect of motion during camera acquisition and synchronization of the camera with PC and power supply. This aforementioned problems are most likely to be encountered during aerial drone imaging of PV plants for diagnostics. Building this setup help us having a detailed understanding of the described problems and finding solutions for it. Measurements are displayed showing the distortion of the images at different tilt angles, with and without varying controlled light noise level displaying the versatility of this setup. The setup will also help to demonstrate proof of concept for the described measurement techniques and will be useful for evaluating the performance of the EL-lock in imaging technique method. As a scope for future work, IRT would be implemented parallely/sequentially along with EL lock-in on the same tripod head having the same setup and system configuration for analysing the information extracted from IR and EL images for failure diagnostics. 


\section{ACKNOWLEDGMENT}

This work was partially supported by Innovation Fund Denmark within the research project DronEL - Fast and accurate inspection of large photovoltaic plants using aerial drone imaging, project number 6154-00012B.

\section{REFERENCES}

[1] M. Kntges, S. Kurtz, C. E. Packard, U. Jahn, K. Berger, K. Kato, T. Friesen, H. Liu, and M. Van Iseghem, Review of Failures of Photovoltaic Modules. 9783906042169, 2014.

[2] O. Breitenstein and C. Schmidt, Thermal failure analysis by IR lock-in thermography, Failure Analysis Desk , pp. 330339, 2004.

[3] S. Dotenco, M. Dalsass, L. Winkler, T. Wurzner, C. Brabec, A. Maier, and F. Gallwitz, Automatic detection and analysis of photovoltaic modules in aerial infrared imagery, 2016 IEEE Winter Conference on Applications of Computer Vision (WACV), pp. 19, 2016.

[4] https://www.alliedvision.com/en/products/cameras/detail/Goldeye /CL-033 TEC1.html.

[5] H. Lawn, Operator S Manual, no. 770.

[6] An Introduction to the Sampling Theorem, National Semiconductor Application Note 236, AN-236,January 1980.

[7] O. Breitenstein, W. Warta, and M. Langenkamp, Lock-in Thermography. 3540210814, 2005.

[8] S. Guide, Keysight True volt Series Digital Multimeters.

[9] C. Mantel, S. Spataru, H. Parikh, D. Sera, G. A. dos Reis Benatto, N.Riedel, S.Thorsteinsson, P.B. Poulsen and S. Forchhammer "Correcting for Perspective distortion in ELectroluminescence Images of Photovoltaic Panels" in Conference: 2018 IEEE 7th World Conference on Photovoltaic Energy Conversion (WCPEC) (A Joint Conference of 45th IEEE PVSC, 28th PVSEC, 34th EU PVSEC) pp. 1-5.

[10] K. bedrich, M. Bliss, T.R. Betts, R.Gottschalg, "EL Imaging of PV Devices: Uncertainty due to Optical and Perspective Distortion" in Conference: 31st European Photovoltaic Solar Energy Conference and Exhibition (EUPVSEC), 2015. pp. 1-6.

[11] G. A. dos Reis Benatto, C. Mantel,A. Santamaria Lancia, N.Riedel, S.Thorsteinsson, P.B. Poulsen, S. Forchhammer, H. Parikh, S. Spataru and D. Sera, "Drone-Based Daylight Electroluminescence Imaging of PV Modules" in Conference: 2019 46th IEEE PVSC Journal of photovoltaics pp. 1-6.

[12] H. Parikh, S. Spataru, C. Mantel, G. A. dos Reis Benatto, D. Sera, "Enhancement of Electroluminescence images for fault detection in photovoltaic panels" in Conference: 2018 IEEE 7th World Conference on Photovoltaic Energy Conversion (WCPEC) (A Joint Conference of 45th IEEE PVSC, 28th PVSEC, 34th EU PVSEC) pp. 1-6.

[13] C. Mantel, G. A. dos Reis Benatto, N. Riedel, P.B. Poulsen, H. Parikh, S. Spataru, D. Sera, S. Forchhammer, "SNR Study of Outdoor Electroluminescence Images under High Sun Irradiation" in Conference: 2018 IEEE 7th World Conference on Photovoltaic Energy Conversion (WCPEC) (A Joint Conference of 45th IEEE PVSC, 28th PVSEC, 34th EU PVSEC) pp. 1-5. 